La forte demande actuelle de nouvelles technologies de I'énergie conduit à des études fondamentales sur des sujets extrêmement appliqués, avec un souhait de réalisation de dispositifs performants.

C'est le cas de l'élaboration des matériaux pour les piles à combustible, convertisseurs d'énergie chimique en source de courant et de chaleur, voire également de produits chimiques à haute valeur ajoutée.

\section{Cet article décrit le couplage} de diverses approches physiques pour comprendre et développer de nouvelles électrodes de piles à combustible, en vue de permettre la réalisation de véhicules à hydrogène à un coût compatible avec une production industrielle.

Remerciements

Nous remercions très vivement le CNRS, qui, au travers de l'INSIS, de l'INC, du programme Énergie et successivement des GdR PACEM, PACTE et PACS, nous a constamment soutenus.

\title{
Physique, Plasmas, Matériaux et Énergie Les piles à combustible
}

Pascal Brault ${ }^{(1)}$ (Pascal.Brault@univ-orleans.fr), Jean-Marc Bauchire ${ }^{(1)}$, Amaël Caillard ${ }^{(1)}$, Anne-Lise Thomann ${ }^{(1)}$, Mathieu Mougenot ${ }^{(1,2)}$, Christophe Coutanceau ${ }^{(3)}$, Steve Baranton ${ }^{(3)}$, Pascal Andreazza $^{(4)}$, Caroline Andreazza-Vignolle ${ }^{(4)}$, François James ${ }^{(5)}$ et Christophe Josserand ${ }^{(6)}$ (1) GREMI, UMR 7344, CNRS-Université d'Orléans, BP 6744, 45067 Orléans Cedex 2

(2) Agence Innovation Made in Dreux, 4 rue Caquot, 28500 Vernouillet

(3) IC2MP, UMR7285, CNRS-Université de Poitiers, 86034 Poitiers Cedex

(4) CRMD, FRE 3520, CNRS-Université d'Orléans, 45071 Orléans Cedex 2

(5) MAPM0, UMR 7349, CNRS-Université d'Orléans, BP 6759, 45067 Orléans Cedex 2

(6) IJLRA, UMR 7190, CNRS-Université Paris 6, 75252 Paris Cedex 05

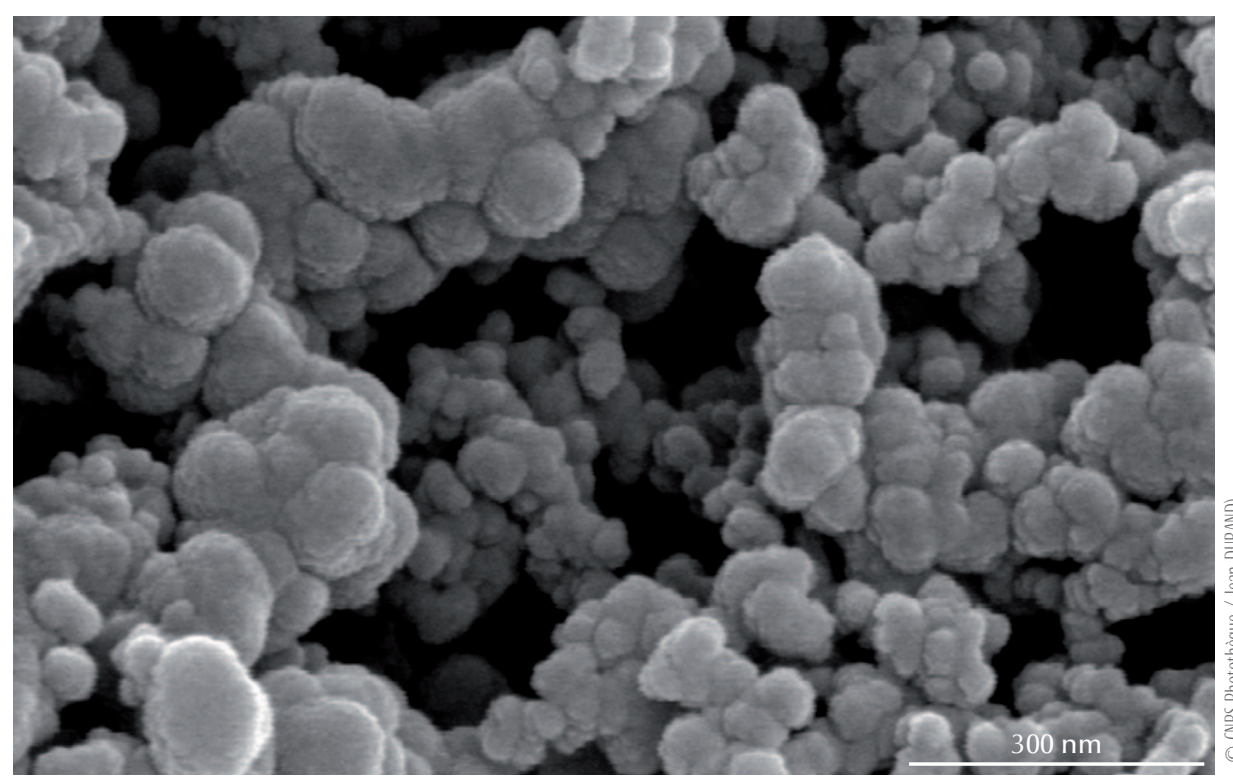

Électrode de piles à combustible PEMFC obtenue par pulvérisation plasma (GREMI, UMR CNRS - Université d'Orléans), vue au microscope électronique à balayage.

\section{Piles à combustible à membrane}

Le principe de base est extrêmement simple : une pile à combustible est composée de deux électrodes (anode et cathode) et d'un électrolyte. Pour simplifier la discussion, nous ne décrirons ici que les piles à électrolyte polymère (appelé membrane) qui peuvent fonctionner à température ambiante, et qui sont des candidates de choix pour les applications transports et nomades (p. ex. téléphones mobiles). Les piles à combustible à membrane échangeuse de protons (dont l'acronyme anglais est PEMFC $=$ Proton Exchange Membrane Fuel Cell) sont les représentantes les plus abouties de cette catégorie. À l'anode, un combustible contenant de l'hydrogène est dissocié catalytiquement (permettant ainsi un fonctionnement à basse température), libérant des protons qui traversent la membrane. Les électrons, également produits à l'anode, circulent dans un circuit extérieur et se recombinent à la cathode avec les protons et l'oxygène qui y est délivré, pour former de l'eau et de la chaleur, la réaction étant exothermique (voir encadré 1).

\section{Pulvérisation plasma}

Le GREMI (Groupe de recherches sur l'énergétique des milieux ionisés) a, depuis quelques années, mis au point une technique de pulvérisation plasma (voir encadré 2, p. 24), permettant de réaliser les électrodes de ces piles à combustible avec de très faibles 
charges de catalyseur [1-4]. Ce dernier point est extrêmement important, car le catalyseur le plus efficace est le platine dont le coût est très élevé : $40 €$ le gramme (février 2012). Pour mesurer l'importance de ce point, il faut savoir que la puissance de pile nécessaire pour un véhicule automobile est d'environ $80 \mathrm{~kW}$. Il faut actuellement $80 \mathrm{~g}$ de platine pour atteindre cette puissance, ce qui conduit à un coût de $3200 €$, uniquement pour le catalyseur. Un tel coût est bien évidemment prohibitif. Pour pallier à cette limitation, plusieurs groupes se sont lancés dans la course à la réduction des quantités de platine. Nous avons ainsi obtenu, en déposant la phase catalytique par pulvérisation plasma, des taux de platine aussi faibles que $10 \mu \mathrm{g}_{\mathrm{Pt}} \cdot \mathrm{cm}^{-2}$ et des densités de puissance de $20 \mathrm{~kW} \cdot \mathrm{g}_{\mathrm{Pt}}{ }^{-1}$, ce qui est bien supérieur à ce qui a été obtenu jusque-là, soit 1 à $10 \mathrm{~kW} \cdot \mathrm{gPt}^{-1}$.

Au-delà de l'obtention de ces performances encourageantes, le dépôt par pulvérisation plasma a permis de s'intéresser à des problèmes très fondamentaux. En effet, le support des catalyseurs est une couche poreuse de carbone conducteur, se présentant sous la forme d'un empilement non compact de sphères de carbone de tailles comprises entre 30 et $100 \mathrm{~nm}$, conduisant à une porosité d'environ $60 \%$ et une taille de pores d'environ $100 \mathrm{~nm}$. L'élaboration de la phase catalytique est alors directement liée au problème de transport/diffusion/ agrégation/croissance en milieu poreux. Pour traiter le problème, nous avons mis en place trois approches: une étude théorique de la «diffusion " du platine dans la couche poreuse $[5,6]$, une étude de la répartition des agrégats dans le milieu poreux par diffusion des rayons $\mathrm{X}$ aux petits angles en incidence rasante (GISAXS), et une étude par microscopie électronique en transmission à haute résolution (HRTEM) [7].

Le principe du dépôt par pulvérisation plasma consiste à délivrer sur un substrat un flux d'atomes provenant d'un matériau cible (encadré 2). Ces atomes ont une fonction de distribution en énergie qui dépend de la pression du plasma et de la distance cible-substrat, ce qui détermine le nombre de collisions sur ce trajet et donc les pertes d'énergie. Pour fixer les idées, à faible pression $(0,5 \mathrm{~Pa})$ et faible distance $(5 \mathrm{~cm})$, l'énergie moyenne des atomes de platine est d'environ $7 \mathrm{eV}$, alors qu'à plus

\section{Qu'est-ce qu'une pile à combustible ?}

encadré 1

Une pile à combustible permet de convertir directement de l'énergie chimique (transformation de réactifs en produits de réaction) en énergie électrique. Comme cela est présenté sur la figure E1, une pile est constituée d'un cœur, formé de deux électrodes séparées par un électrolyte, et de dispositifs garantissant l'approvisionnement en combustible et en comburant, et l'évacuation des produits de réaction.

Dans une PEMFC, le combustible hydrogène est introduit côté anode, puis dissocié à I'interface anode-membrane suivant la réaction électrochimique $\mathrm{H}_{2} \rightarrow 2 \mathrm{H}^{+}+2 \mathrm{e}^{-}$, qui fait intervenir le catalyseur (Pt). Les ions $\mathrm{H}^{+}$diffusent dans l'électrolyte jusqu'à la cathode. Les électrons sont contraints de se déplacer dans l'électrode (carbone), puis dans un circuit extérieur, créant un courant continu. Les électrons, les protons et l'oxygène se retrouvent à l'interface cathode-membrane pour former de l'eau. La réaction s'accompagne d'un dégagement de chaleur, qui peut être récupéré. Le bilan global est : hydrogène + oxygène $\rightarrow$ eau + électricité + chaleur, avec une efficacité supérieure à 90\%.

Les réactions électrochimiques n'ont lieu que dans les zones de triple contact entre le platine, le carbone (éléments constituant les électrodes) et la membrane (fig. E2).

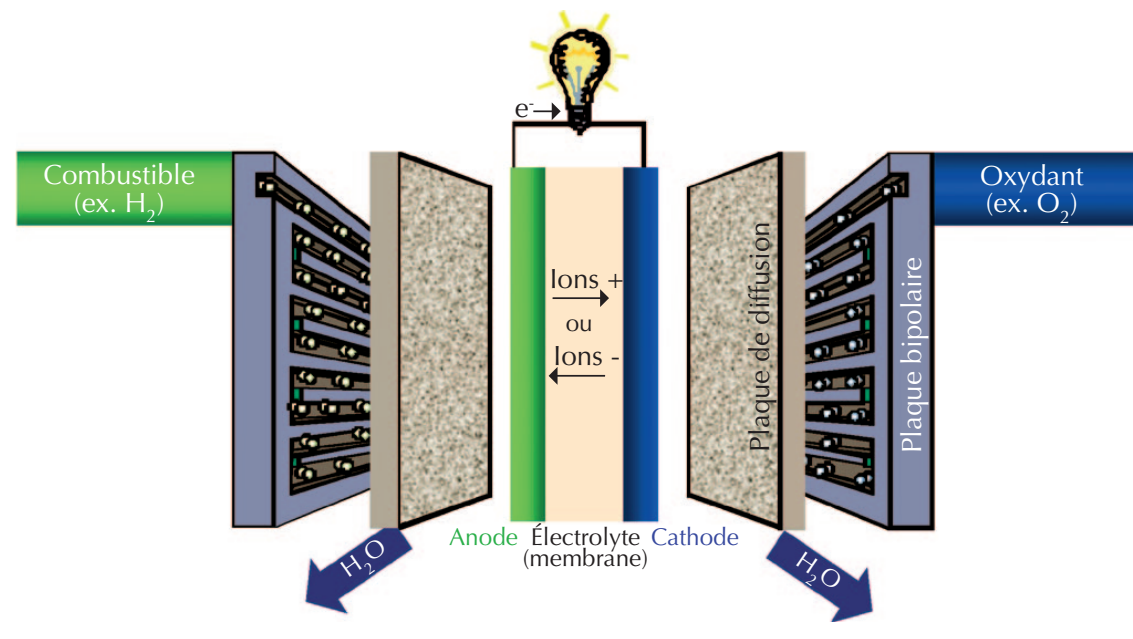

E1. Schéma d'une PEMFC $\mathrm{H}_{2} / \mathbf{0}_{2}$. Les principaux éléments sont

(a) l'électrolyte, en général fine membrane polymère solide (nafion ${ }^{\odot}$ ), qui doit conduire les protons, mais pas les électrons, et être imperméable aux gaz ;

(b) les électrodes (anode et cathode), constituées d'un métal catalyseur (Pt) déposé sur une couche poreuse de carbone conducteur ;

(c) les plaques de diffusion :

(d) les plaques bipolaires, parcourues de microcanaux qui permettent d'assurer la distribution des gaz et l'évacuation de l'eau.

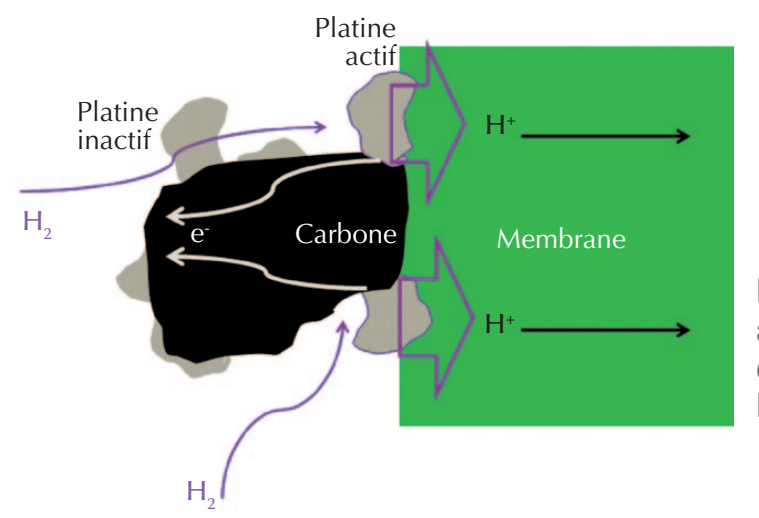

E2. Localisation des points triples à l'interface entre la couche catalytique et la membrane d'une PEMFC. 


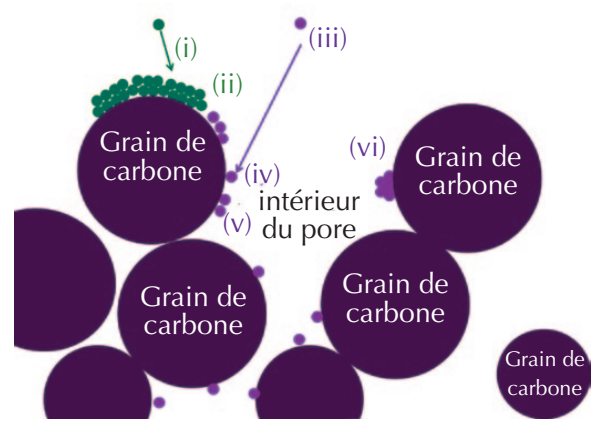

1. Illustration des processus physiques intervenant dans la séquence dépôt/transport/diffusion/ croissance dans un pore issu de l'empilement de grains de carbone.

(i) Adsorption et croissance sur le haut d'une sphère ; (ii) diffusion du haut d'une sphère vers la paroi interne d'un pore ;

(iii) transport/adsorption directement sur une paroi interne de pore :

(iv) diffusion le long de la paroi ;

(v) nucléation d'un agrégat ;

(vi) croissance d'un agrégat supporté dans un pore. Ce schéma peut s'étendre simplement à toute configuration/géométrie de pores ouverts $[2,6]$.

\section{Qu'est-ce que} la pulvérisation plasma?

Un plasma d'argon est créé dans une enceinte sous vide. Une cible est polarisée négativement, et les ions positifs $\mathrm{Ar}^{+}$situés à proximité de la cible sont attirés par la chute de potentiel. En interagissant avec la surface, ils pulvérisent les atomes de la surface à la suite d'une série de collisions. Les atomes pulvérisés sont libérés de la cible, traversent le plasma (transport plus ou moins collisionnel suivant la pression du plasma) et se déposent sur un substrat placé en vis-à-vis, comme le représente la figure E3.

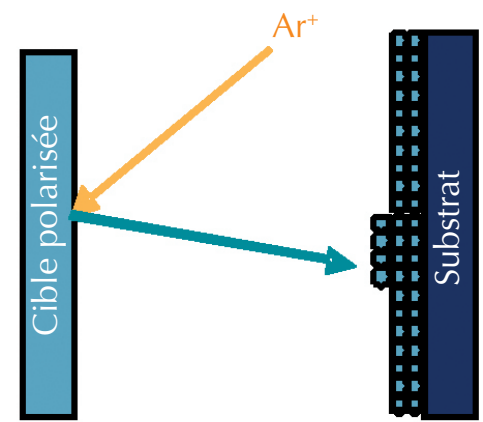

B. Schéma de principe de la pulvérisation plasma.

\section{>>}

haute pression l'énergie cinétique peut être thermalisée, soit $0,03 \mathrm{eV}$. Ce flux d'atomes pulvérisés va donc interagir avec le substrat poreux selon de multiples possibilités, comme le schématise la figure 1.

\section{Diffusion du catalyseur sur un substrat poreux}

L'exposition d'un milieu poreux à un flux d'atomes conduit à une pénétration plus ou moins grande de ces atomes à l'intérieur du milieu, comme le montre la figure 1. Les mesures par spectroscopie de rétrodiffusion de Rutherford menées sur l'accélérateur Van de Graaff du CEMHTI (UPR 3079 CNRS, Orléans), ont permis de déterminer les profils de concentration de platine dans la profondeur de la couche poreuse par ajustement aux moindres carrés entre les spectres expérimentaux et les spectres simulés à partir de compositions d'essai. Comme cela était attendu, ces profils sont décroissants de la surface vers l'intérieur de la couche. Il était intéressant de vérifier s'ils obéissaient, ou non, à une équation de diffusion de la matière. La littérature est riche de la description de phénomènes de transport/diffusion. Nous nous sommes donc penchés sur ceux concernant les milieux poreux, et nous avons testé l'équation de diffusion unidimensionnelle correspondante $[5,6]$ :

$\partial \rho / \partial t=\partial[K(z, t) \partial \rho / \partial z)] / \partial z \quad(1)$,

où $\rho(z, t)$ est la concentration en fonction de la profondeur au temps $t$. Le coefficient de diffusion prend la forme :

$K(z, t)=K_{0} t^{\gamma} / z^{\theta}$, où $K_{0}$ est une constante. Les paramètres $\theta$ et $\gamma$ prennent en compte le fait que la morphologie du substrat poreux peut évoluer à mesure que la quantité de platine y augmente, à la fois dans l'espace et dans le temps. De plus, le flux arrivant à la surface du substrat $(\mathrm{z}=0)$ est supposé dépendre du temps $\left(\propto t^{\varepsilon}\right)$, du fait du changement de morphologie pendant le dépôt.

Tableau 1. Exposants $\theta, \beta, \gamma$ et $\varepsilon$ pour trois conditions différentes de pulvérisation plasma : C1, C2 et AAO. C1 et C2 correspondent au même substrat, un empilement de sphères de carbone, dans des conditions de pression d'argon différentes $\left(\mathrm{p}^{\mathrm{C} 1}{ }_{\mathrm{Ar}}<\mathrm{p}^{\mathrm{C} 2}{ }_{\mathrm{Ar}}\right)$. Pour AAO, le substrat est une matrice d'alumine traversée par un réseau hexagonal de pores nanométriques cylindriques (de diamètre $100 \mathrm{~nm}$ ), dans les mêmes conditions plasma que C1.
Ceci conduit alors à un profil de concentration [5] en fonction de la profondeur :

$$
\rho(z, t) \propto t^{\beta} \exp \left[-\frac{(1+\gamma) z^{2+\theta}}{(2+\theta)^{2} K_{0} t^{1+\gamma}}\right]
$$

L'exposant $\beta$ est une fonction des paramètres $\varepsilon, \gamma$ et $\theta$. $\theta$ dépend plutôt des conditions plasma, alors que $\varepsilon$ et $\gamma$ sont plutôt fonction du matériau poreux.

Dans le tableau 1 sont reportées les valeurs de ces paramètres pour différentes conditions d'élaboration. C1 et C2 représentent des dépôts sur des empilements de grains de carbone de taille 30-100 nm (comme schématiquement représenté sur la figure 1), pour deux pressions d'argon différentes [5], alors que AAO correspond à un dépôt à la même pression que C1, mais dans un réseau hexagonal de trous cylindriques de diamètre $100 \mathrm{~nm}$ d'un substrat d'oxyde d'aluminium anodisé [6]. $\varepsilon<0$ (cas du substrat de carbone) signifie que le flux entrant dans le matériau poreux décroît dans le temps et est limité du fait de la croissance de la surcouche (processus (i), figure 1); alors que $\varepsilon>0$ (cas du substrat d'alumine) correspond au processus (ii) où les atomes de la surcouche contribuent à la "diffusion » sur les parois du pore. $\gamma<0$ correspond à un coefficient de diffusion qui diminue au cours du temps, par exemple du fait du rétrécissement des pores, tandis que $\gamma>0$ décrit une diffusion accélérée.

Les différences entre les deux milieux sont la plus faible porosité et l'absence de tortuosité pour le support AAO. Malgré cette plus faible porosité, on constate que la diffusion est exaltée dans ce dernier cas $(\varepsilon>0, \gamma>0)$. Ceci peut s'expliquer par le fait que l'absence de tortuosité rend le transport dans le pore cylindrique plus efficace que dans l'empilement de sphères, qui pourtant présente une plus forte porosité, mais une plus faible accessibilité. On

\begin{tabular}{c|c|c|c|c|}
\hline Conditions & $\boldsymbol{\theta}$ & $\boldsymbol{\beta}$ & $\boldsymbol{\gamma}$ & $\boldsymbol{\varepsilon}$ \\
\hline $\mathrm{C} 1$ & $-1,50$ & 0.40 & $-0,60$ & $-0,20$ \\
\hline $\mathrm{C} 2$ & $-1,33$ & 0.25 & -0.75 & $-0,25$ \\
\hline AAO & $-1,66$ & 0,75 & 0,25 & 4,00 \\
\hline
\end{tabular}


atteint là les limites du modèle proposé, qui ne tient compte ni de la géométrie du support matériel, ni des processus physiques mis en jeu (couplage transport-adsorption).

\section{Croissance des agrégats}

L'un des points importants pour l'électrocatalyse dans les piles à combustible est la maîtrise du processus de croissance des agrégats de catalyseur. En effet, la taille et la morphologie des particules métalliques sont des paramètres importants de l'activité (vitesse de réaction aux électrodes) et de la sélectivité (production d'eau à la cathode) des matériaux catalytiques [8]. Pour comprendre comment la pulvérisation plasma peut permettre le dépôt/croissance d'agrégats appropriés pour les électrodes de piles à combustible, nous avons mené une étude conjointe de microscopie électronique en transmission et de diffusion de rayons $\mathrm{X}$ aux petits angles en incidence rasante (GISAXS, voir encadré 3) [9, 10].

Pour cette étude, nous avons réalisé entièrement les électrodes par pulvérisation plasma, en codéposant simultanément le platine et le carbone. En fait, cette configuration a permis d'obtenir les performances en pile très intéressantes citées plus haut [3]. Il était donc pertinent de comprendre la croissance des agrégats de platine lors du processus de copulvérisation.

La copulvérisation conduit à une croissance en colonne mixte carbone-platine, observée sur la figure 2 où les zones les plus claires correspondent à la présence de platine. On constate un évasement des colonnes vers la surface du dépôt (haut de la figure). Ceci correspond aux effets d'ombrage inhérents à la croissance colonnaire. Il est cependant difficile de savoir si du platine se trouve à l'intérieur des colonnes.

Par rapport à la microscopie électronique à balayage, de moins bonne résolution, et au GISAXS plus statistique, la microscopie électronique en transmission donne des informations plus précises et plus locales quant à la nature des agrégats de platine, comme cela peut se voir sur la figure 3 .

La figure $3 \mathrm{~b}$ montre distinctement que les agrégats de platine s'arrangent en lignes perpendiculaires à l'axe de croissance de la colonne, et que la taille de ces agrégats est de l'ordre de $2 \mathrm{~nm}$. Cependant, il est difficile d'attribuer à ces résultats une portée statistique. C'est pour cela qu'il a été nécessaire de les confirmer et les généraliser par une étude GISAXS (cf. encadré 3), qui

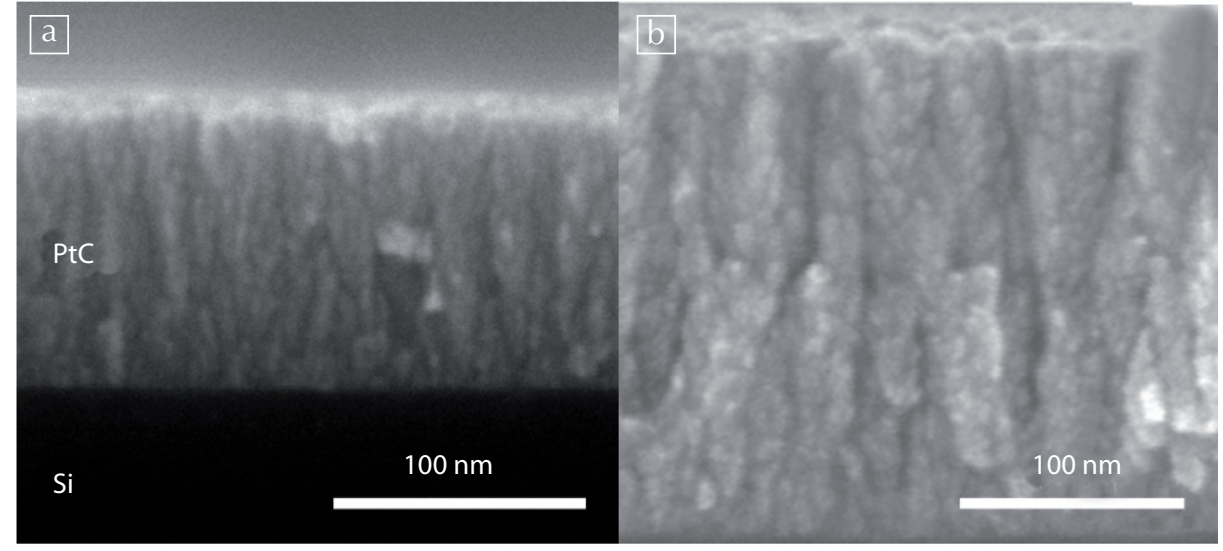

2. Clichés de microscopie électronique à balayage de couches carbone-platine déposées sur un substrat de silicium, révélant une structure colonnaire de la couche.

(a) Couche de 110 nm d'épaisseur. (b) Couche de 300 nm d'épaisseur.

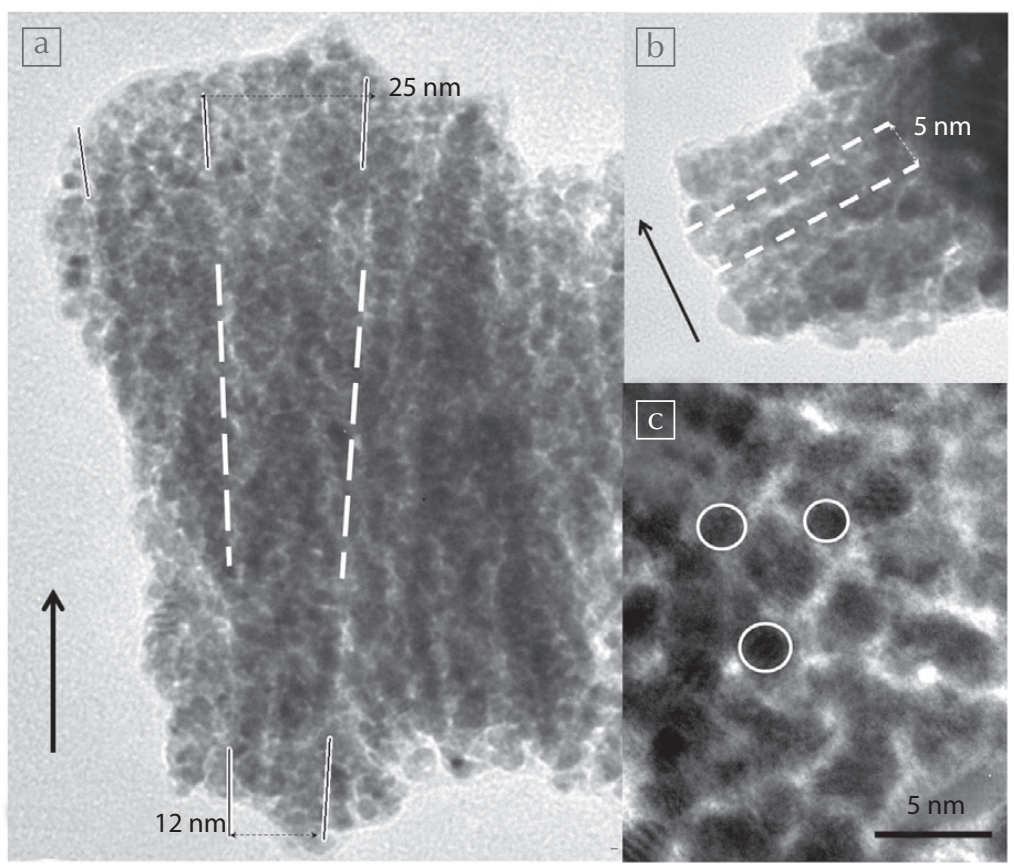

3. Clichés de microscopie électronique en transmission sur une coupe transverse d'une couche carboneplatine. Les agrégats de platine sont en noir. Les flèches indiquent la direction de croissance.

a) Structure colonnaire de carbone comprenant les agrégats de platine. Les tirets blancs montrent l'évasement des colonnes. b) Zoom avec les tirets blancs indiquant la distance entre deux colliers de platine successifs.

c) Zoom avec les cercles blancs délimitant des agrégats de Pt.

Tableau 2. Détermination des tailles des agrégats de platine et des distances interagrégats, parallèlement et perpendiculairement au plan de croissance.

\begin{tabular}{|c|c|c|c|c|c}
\hline \multirow{2}{*}{ PtC20 } & $\alpha_{i}$ & $D_{x y}(n m)$ & $\Lambda_{x y}(n m)$ & $D_{z}(n m)$ & $\Lambda_{z}(n m)$ \\
\cline { 2 - 6 } & $0,28^{\circ}$ & 1,6 & 2,9 & 2,2 & 4,2 \\
\hline \multirow{2}{*}{ PtC60 } & $0,35^{\circ}$ & 1,6 & 2,1 & 2,2 & 4,7 \\
\cline { 2 - 6 } & $0,37^{\circ}$ & 1,6 & 2,6 & 2,0 & 4,8 \\
\hline \multirow{2}{*}{ PtC100 } & $0,51^{\circ}$ & 1,6 & 1,8 & 2,0 & 4,2 \\
\cline { 2 - 6 } & $0,27^{\circ}$ & 1,6 & 3,2 & 2.2 & 4,8 \\
\cline { 2 - 6 } & $0,33^{\circ}$ & 1,6 & 2,9 & 2,0 & 5,1 \\
\hline
\end{tabular}




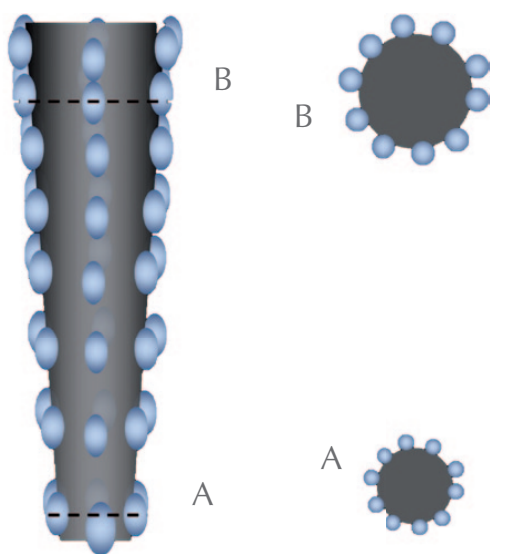

4. Représentation de la structure PtC colonnaire moyenne, obtenue par pulvérisation magnétron simultanée de platine et de carbone. Les agrégats de platine (en bleu) forment des colliers en périphérie des colonnes de carbone (en noir).

\section{\>}

permet d'obtenir des valeurs moyennes des tailles d'agrégats (D) et distances interagrégats $(\Lambda)$, parallèlement (indices xy) et perpendiculairement (indice z) au plan de croissance. Les mesures ont été effectuées sur la ligne SWING du synchrotron SOLEIL, sur trois échantillons, PtC20, PtC60 et PtC100, d'épaisseurs respectives 20, 60 et $100 \mathrm{~nm}$, à incidence $\alpha_{i}$ variable du rayonnement $\mathrm{X}$ pour sonder des profondeurs différentes [7]. Ainsi, les caractéristiques des agrégats en fonction de la profondeur ont pu être déterminées. Les résultats, reportés sur le tableau 2, sont cohérents avec ceux des observations par microscopie électronique.
Les distances interagrégats, parallèlement au plan de croissance $\left(\Lambda_{\mathrm{xy}}\right)$, augmentent lorsque $\alpha_{i}$ décroît, c'est-à-dire lorsque l'on se rapproche de la surface, ce qui est cohérent avec la forme évasée des colonnes supportant les agrégats de platine. De même, les distances perpendiculaires au plan de croissance $\left(\Lambda_{z}\right)$ diminuent lorsque l'on se rapproche de la surface. Ceci indique une organisation en colliers autour des colonnes. Les colliers d'une colonne se rapprochent près de la surface. La distance interagrégats dans un collier est plus grande près de la surface. D'autre part, le diamètre des agrégats parallèlement au plan de croissance $\left(D_{x y}\right)$ est plus petit que celui mesuré dans l'axe des colonnes : $\mathrm{D}_{\mathrm{z}}>\mathrm{D}_{\mathrm{xy}}$. Nous avons donc des agrégats oblongs le long de l'axe des colonnes. Ceci est cohérent avec un flux de dépôt parallèle à l'axe de croissance. La figure 4 résume le mode de croissance du platine copulvérisé avec le carbone.

\section{Conclusion}

Ces travaux ont permis de mettre en évidence les mécanismes de transport/ diffusion du platine dans différentes couches poreuses, et de comprendre comment le plasma pouvait être responsable de la pénétration de platine dans ces couches. D'autre part, le mode de croissance des agrégats lors de la copulvérisation plasma a pu être déterminé : la croissance des agrégats se fait en décorant les colonnes de carbone. Le contrôle de la valeur des flux de platine et de carbone permet d'ajuster les distances interagrégats à l'évasement des colonnes.

Au-delà de la compréhension des mécanismes de croissance du catalyseur, ces travaux ont conduit à améliorer les performances des piles à combustible utilisant des électrodes ayant une couche active catalytique composée d'agrégats de platine supportée sur des particules de carbone. Ceci est largement dû au fait que la pulvérisation plasma permet de contrôler des profils de concentration de catalyseur dans les électrodes pertinents pour un fonctionnement optimal des piles, tout en réduisant considérablement la quantité de ces catalyseurs très coûteux. En effet, le gain de performance est d'un facteur 10 en terme de coût de matière : passage de 0,1 à $0,01 \mathrm{mg}_{\mathrm{Pt}} \cdot \mathrm{cm}^{-2}$, pour des puissances délivrées comparables à l'existant. Cette réduction de coût rend plus réaliste l'utilisation de PEMFC à catalyseur de platine dans les véhicules à hydrogène.

Les futurs travaux concernent maintenant le contrôle du vieillissement de ces électrodes, ce qui nécessite la prévention de la coalescence des agrégats de platine durant le fonctionnement en pile. L'idéal sera bien sûr de trouver des solutions sans catalyseurs à base de métaux nobles. Un tel objectif fait actuellement l'objet de recherches intensives.

\section{Qu'est-ce que la diffusion des rayons $X$} aux petits angles en incidence rasante ?

encadré 3

La technique GISAXS (Grazing Incidence Small Angle X-ray Scattering) permet de caractériser les hétérogénéités de densité dans les zones superficielles d'un matériau, telles que des particules dans une matrice. Les mesures sont réalisées sous des angles d'incidence $\alpha_{i}$ variables $\left(0,2^{\circ}-0,6^{\circ}\right)$, proches de l'angle critique de réflexion totale de la couche mince à analyser, permettant de sonder des zones plus ou moins profondes de la couche. Les rayons diffusés sont observés avec des angles d'émergence $\alpha_{f}$ faibles (petits angles). L'analyse fine de la figure de diffusion (répartition de l'intensité diffusée dans le plan perpendiculaire au faisceau incident) permet de déduire la forme, l'orientation, la taille et la distribution moyenne des objets diffusants présents dans la couche mince (voir G. Renaud, Reflets de la physique 34-35 (2013) 65-69).

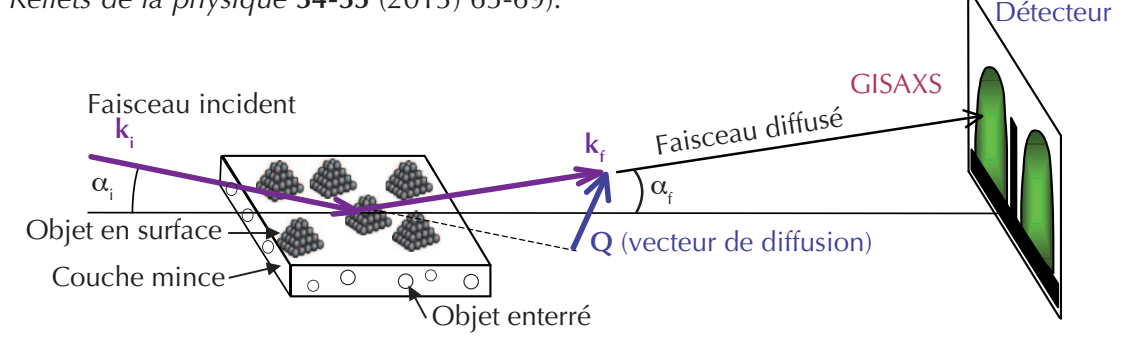

E4. Principe de la technique GISAXS.

\section{Références}

1• P. Brault et al., J. Phys. D, 37 (2004) 3419

2• A. Caillard et al., Appl. Phys. Lett., 90 (2007) 223119.

3• M. Cavarroc et al., Electrochem. Comm., 11 (2009) 859.

4・ M. Mougenot et al., Int. J. Hydrogen Energy, 36 (2011) 8429

5• P. Brault et al., Phys. Rev. Lett., 102 (2009) 045901

6• S. Wu et al., Physica A, 390 (2011) 2112.

7• M. Mougenot et al., J. Nanoparticle Research, 14 (2012) 672 .

$8 \bullet$ C. Coutanceau et al., "Platinum Fuel cell nanoparticle syntheses: effect on morphology, structure and electrocatalytic behavior" in Nanoparticles / Book 2, InTech Open Access Publisher, University Campus, STeP Ri Slavka Krautzeka 83/A, Rijeka, Croatia, 2011. ISBN 979-953-307-859-4.

9• J. Penuelas et al., Phys. Rev. Lett., 100 (2008) 115502.

10• P. Andreazza, "Probing nanoalloys structure and morphology by X-ray scattering and absorption methods", dans Nanoalloys, Ed. D. Alloyeau, C. Mottet, C. Ricolleau (Springer, 2012). 